\title{
WHAT DO SOLAR F-MODE FREQUENCIES TELL US?
}

\author{
H. M. ANTIA AND S. M. CHITRE \\ Tata Institute of Fundamental Research \\ Homi Bhabha Road, Mumbai 400005, India
}

The fundamental mode (f-mode) which is essentially a surface mode can be expected to provide a diagnostic of flows and magnetic fields etc. present in the surface regions (Ghosh, Chitre \& Antia 1995; Rosenthal \& Christensen-Dalsgaard 1995). It turns out that the observed f-mode frequencies can also provide an accurate measure of solar radius (Antia 1997; Schou et al. 1997).

The frequencies of $\mathrm{f}$-modes are asymptotically expected to satisfy the simple dispersion relation, $\omega^{2}=g k$, where $g$ is the acceleration due to gravity at the surface and $k=\sqrt{\ell(\ell+1)} / r$ is the horizontal wave number. At the moderate values of degree $\ell$ for which the frequencies have been measured, the f-modes are effectively localized somewhat below the solar surface, where $g k$ would be larger than its value at the solar surface. As a result, there is a small departure from this simple dispersion relation. In order to account for this difference we take the ratio of the observed frequencies and those computed for a solar model, and the results are shown in Figure 1. It is clear that this ratio is more or less constant within the expected errors even when $\ell$ varies by more than a factor of two. In order to explain the observed discrepancy the solar radius will need to be decreased by about $0.03 \%$ or about $210 \mathrm{~km}$, which is perhaps somewhat larger than the quoted uncertainty of $70 \mathrm{~km}$ in the solar radius.

The frequencies of $\mathrm{f}$-modes will also be affected by other factors such as the density stratification in the outer layers of the Sun, the surface hydrogen abundance, atmospheric opacities and treatment of convection. For estimating this effects we have constructed different solar models with the revised solar radius estimate of 695.78 Mm. Thus Model M1 uses the CM prescription (Canuto \& Mazzitelli 1991), while Model M2 is based on the usual mixing-length prescription, both using the atmospheric opacity tables of Kurucz (1991). Model M3 is based on the OPAL opacities (Iglesias \& Rogers 1996) and Model M4 which does not include diffusion of elements has a lower hydrogen abundance $X$ and a shallower convection zone. Figure 2 shows the relative difference in frequencies between observed (MDI) values and those for different solar models. We expect the difference $\sim 10^{-4}$ between frequencies of various solar models to be reflected in the estimated solar radius. Thus, if model M2 with MLT treatment of convection is accepted the radius will need to be decreased by about $0.038 \%(265 \mathrm{~km})$, while if model M3 is taken as standard then the radius will need to be reduced by about $0.041 \%(285 \mathrm{~km})$ from the standard value. In general, depending on the treatment of surface layers in the model, the radius needs to be decreased by $200-300 \mathrm{~km}$ over the standard value to match the $\mathrm{f}$-mode frequencies. 


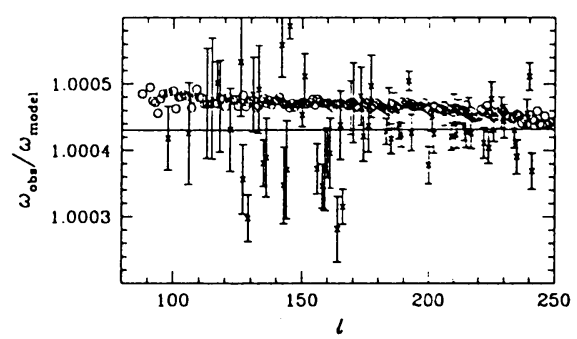

Figure 1. The ratio of observed and model frequencies for f-modes. The crosses with error bars represent the GONG data, while circles represent the MDI data. The horizontal line defines the average over all modes for the GONG data.

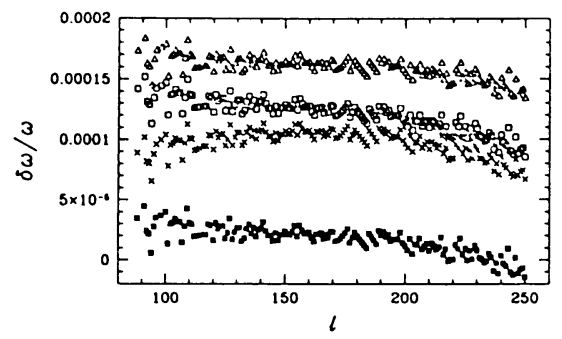

Figure 2. The relative difference in frequencies of $f$-modes between the observed (MDI) values and those of different solar models, with a radius of $695.78 \mathrm{Mm}$. The filled squares, open squares, triangles and crosses respectively, mark the difference for Models M1, M2, M3 and M4.

Further, the lower hydrogen abundance in model M4 also gives rise to a small dip at the lower frequency end in the frequency differences.

When better data on the $f$-mode frequencies at higher $\ell$ become available, it may be possible to separate out contributions from various sources and estimate the value of solar radius more accurately. Since the frequencies of these modes can be determined to a relative accuracy of $10^{-5}$, in principle, it would be possible to determine the solar radius much more accurately. The effects arising from different treatment of surface layers are unlikely to change with solar cycle, and any possible temporal variation in solar radius may be reliably determined via the variation in $\mathrm{f}$-mode frequencies with time.

It may be noted that most of the current standard solar models (e.g., ChristensenDalsgaard et al. 1996) use the value of solar radius as $695.99 \mathrm{Mm}$ with the surface defined at a level where the optical depth is between 1 and $1 / 3$. Clearly, there is a need to revise these models with a possible reduction in the value of solar radius by about 200-300 km. Likewise, most helioseismic inversions that assume a similar definition of solar radius will also need to be revised. In order to estimate the possible errors arising from uncertainty in the value of solar radius, we have attempted helioseismic inversions for the sound speed using the GONG months 4-10 data with different estimates of radius. It appears that the difference caused due to a change of solar radius by $210 \mathrm{~km}$, is much more than the estimated errors in helioseismic inversions over most of the solar interior (Antia 1997). Clearly, we need a reliable measure of the solar radius for inferring conditions in the solar interior with sufficient accuracy.

\section{References}

Antia, H. M. 1997, A\&A (in press) astro-ph/9707226

Canuto, V. M., \& Mazzitelli, I. 1991, ApJ 370, 295

Christensen-Dalsgaard, J., Däppen, W. et al. 1996, Science 272, 1286.

Ghosh, P., Chitre, S. M. \& Antia, H. M. 1995, ApJ 451, 851

Iglesias, C. A. \& Rogers, F. J. 1996, ApJ 464, 943

Kurucz, R. L., 1991, In Stellar atmospheres: beyond classical models, ed. L. Crivellari, I.

Hubeny, D. G. Hummer, NATO ASI Series (Dordrecht: Kluwer), 441

Rosenthal, C. S. \& Christensen-Dalsgaard, J. 1995, MNRAS 276, 1003

Schou J., Kosovichev A. G., Goode P. R., Dziembowski W. A., 1997, ApJL (in press) 